\title{
An Energy-efficient Localization System for Imprecisely Positioned Sensor Nodes with Flying UAVs
}

\section{Conference Paper}

\section{Author(s):}

Niculescu, Vlad; Magno, Michele; Palossi, Daniele (D); Benini, Luca (D)

Publication date:

2020-07

Permanent link:

https://doi.org/10.3929/ethz-b-000461445

Rights / license:

In Copyright - Non-Commercial Use Permitted

Originally published in:

https://doi.org/10.1109/INDIN45582.2020.9442075

Funding acknowledgement:

190880 - 5liber Learning-UAV: Artificial Intelligence-based Ultra-tiny UAVs (SNF) 


\title{
An Energy-efficient Localization System for Imprecisely Positioned Sensor Nodes with Flying UAVs
}

\author{
Vlad Niculescu, Michele Magno, Daniele Palossi, and Luca Benini
}

\begin{abstract}
This work investigates the capability of unmanned aerial vehicles (UAVs) to find and communicate with wireless sensor nodes positioned at unknown locations. In this scenario, the UAV acts as a mobile gateway that estimates the sensor node position using multiple ultra-wideband (UWB) range measurements, before flying in its vicinity to perform energy-efficient data acquisition. In addition to $U W B$, we use wake-up radio (WUR) to improve the sensor node's energy efficiency, keeping it in the always-on "low-activity" state when the drone is not nearby. The paper proposes a localization algorithm that consists of an iterative, noise-robust and computationally lightweight approach based on multi-lateration. Experimental evaluations performed on synthetic data demonstrate that our approach achieves a submeter localization accuracy using only three range measurements. We confirm this with an extensive in-field evaluation. The multilateration algorithm runs in $4 \mathrm{~ms}$, in low power microcontrollers such as the ARM Cortex-M4F. The WUR and our energy-efficient algorithm enable the sensor node to consume only $31 \mathrm{~mJ}$ during the whole localization-acquisition process. Our solution can be introduced in many other industrial applications where a mobile robot needs to estimate the location of imprecisely positioned objects.
\end{abstract}

Index Terms-UWB, UAV, multi-lateration, energy-efficiency

\section{INTRODUCTION}

In the last few years, unmanned aerial vehicles (UAVs) have been introduced in a wide range of industrial applications, such as aerial inspection and ambient awareness [1], [2], [3].

UAVs can facilitate the operations of an industrial wireless sensor network (WSN) without any expensive additional infrastructure which is not feasible in many scenarios [4]. Remote monitoring represents an industrial application where WSNs play an important role [5], [6], [7]. WSNs consist of a large number of battery-powered wireless sensor nodes that are deployed in a specific area [5], [6]. In many WSN deployments, a local-host is in charge of the data acquisition and it forwards the information to a central infrastructure that is not necessarily placed in the vicinity of the deployment area [8], [9]. UAVs represent an alternative solution to the conventional gateways, overcoming their limits, such as limited bandwidth or infrastructure needs [5], [4].

Vlad Niculescu, Daniele Palossi, and Luca Benini are with the IIS D-ITET, ETH Zürich, emails: vladn@iis.ee.ethz.ch, dpalossi@iis.ee.ethz.ch, and lbenini@iis.ee.ethz.ch. Michele Magno is with the PBL - D-ITET, ETH Zürich, email: michele.magno@pbl.ee.ethz.ch. Luca Benini is also with the DEI, University of Bologna, email: luca.benini@unibo.it.

This work was partially funded by the Swiss National Science Foundation (SNSF) projects: AeroSense (40B2-0_1187087), APROVIS3D (20CH21_1186991), and the 5liber Learning-UAV SNSF Spark grant (190880).

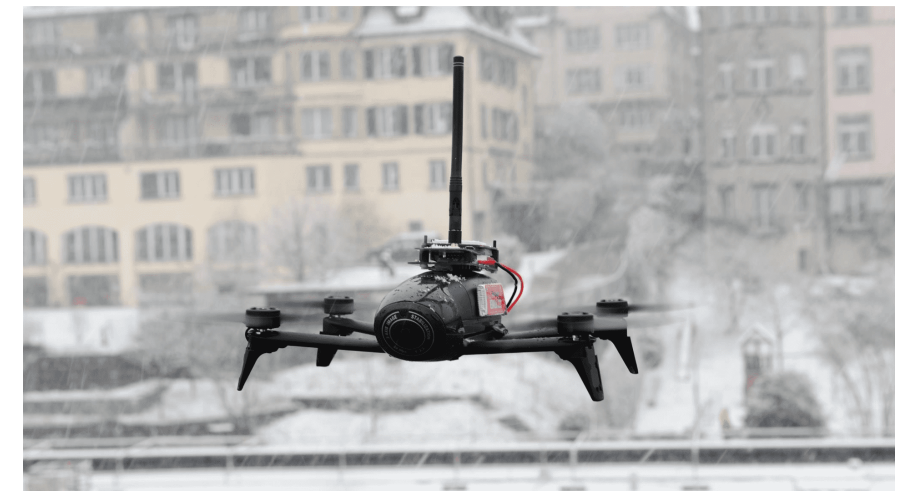

Fig. 1: Our prototype based on the COTS Parrot Bebop 2 quadrotor extended with our UWB-based localization unit.

Due to the UAVs capability to fly to any point in a given area, they enable the nodes of a WSN to operate at a lower transmission power/range [4]. However, in a broad range of industrial applications, the nodes' positions are not a priori known [8], [4], [10], and therefore accurate and fast localization is necessary [5], [8].

Ultra-wideband (UWB) is a widely used ranging technology which allows for accurate distance measurements [11], but it has a high power consumption during the ranging phase (i.e., hundreds of $\mathrm{mW}$ [11]). We use the UWB in combination with a wake-up radio (WUR), a $\mu \mathrm{W}$ always-on receiver [12], to achieve high energy efficiency on the nodes, by keeping the UWB active only when the drone comes close.

Multi-lateration is a navigation technique, which uses at least three distance measurements from fixed and known positions (i.e., anchors) to estimate the location of a moving object. However, in contrast to this conventional scenario [13], this work addresses the dual problem, where the UAV behaves like a moving anchor [14], [15] to estimate the position of the nodes imprecisely deployed in its surrounding.

The main contribution of this paper is the investigation and experimental evaluation of an energy-efficient localization system that enables a UAV to localize unknown sensors within a WSN. More in detail, our work provides the following contributions:

- we developed an embedded multi-lateration algorithm that enables the UAV to precisely estimate the sensor node position while keeping the sensor node energy consumption within $31 \mathrm{~mJ}$. Thus, we combine for the 
first time UWB and WUR for precise and energy-efficient localization;

- we investigated the influence of the number of range measurements on the localization accuracy. We demonstrate that with only three UWB measurements, our algorithm achieves already $1 \mathrm{~m}$ accuracy with the best trade-off with energy consumption;

- we propose the electronic design of both a sensor node that includes a WUR and of an MCU-based "companion board", which operates aboard a commercial-of-the-shelf (COTS) Parrot Bebop 2 quadrotor;

- our in-field evaluation shows that the proposed trilateration algorithm is up to $50 \%$ more accurate than State-of-the-Art (SoA) solutions [16], and it runs in a low power embedded platform in $4 \mathrm{~ms}$.

\section{RELATED WORK}

Recent literature has shown an increasing interest in the combination of UAVs with WSNs for industrial applications [5], [17], [10]. In particular, many approaches have shown the importance of UAVs to perform localization of WSN's nodes to reduce the cost/power of long-range communications [5], [4]. In the majority of these approaches, UAVs are using a combination of sensors [9]. However, cameras can suffer from occlusions and poor lighting conditions that can prevent precise localization in several scenarios, such as dark or cluttered environments. Other popular approaches are range-based algorithms that use measurements of the distance from the drone to the node to calculate the sensor node position [16], [4]. There are several technologies to estimate the range, for instance, received signal strength indicator (RSSI) [8], [18]. Among others, Wifi and Bluetooth are often mentioned as trendy choices [19], [20] due to their vast availability and reduced price. Bluetooth low energy (BLE) is a widely used technology in the context of low power WSNs because it presents a power consumption of few tens of $\mathrm{mW}$ [21]. However, its precision when performing distance measurements is about $1 \mathrm{~m}$, which leads to a localization error of about $2 \mathrm{~m}$ when more than 100 measurements are used from 7 anchors [22].

In contrast to these approaches, the novel UWB ranging method that we use is Time of Arrival (ToA), which determines the range based on the round trip time of the UWB signal, achieving centimeter accuracy [14] in line-of-sight conditions. However, the ranging precision does not imply the same localization precision. The latter strongly depends on where the range measurements are acquired. Furthermore, the uncertainty in the drone position introduces additional errors in estimating the node position. In our work, considering all these real-world effects we obtain a sub-meter precision for the node position [13].

Multi-lateration is a method to determine the location of an object by knowing its distance to other known positions in space and its application to UWB measurements is widely investigated in literature [23], [24], [25], [13]. In [23] it is proposed a range-based multi-lateral measurement localization method (RB-MML). Their approach shows the benefits of using a multi-lateration algorithm in the localization with UWB as we are proposing in our paper. On the other hand, the RR-MML is designed for ideal, noise-free situations and therefore it performs poorly in the presence of real-world noise. [25] proposes an approach similar to ours - using a sophisticated cost function to penalize the error. However, their cost function is not guaranteed to converge to the maximum likelihood solution in the presence of Gaussian noise on the input data, as well as it requires more operations and has a heavier memory footprint compared to our solution.

In [16], the authors also investigate the scenario of a UAV localizing sensor nodes as in our application scenario. They propose a strategy to bound the localization error that is comparable with ours, but they did not deployed it on an embedded demonstrator and their results only rely on postprocessed data in a conventional computer. Furthermore, to achieve a $1 \mathrm{~m}$ accuracy, their UAV has to fly a circular arc of $80^{\circ}$ around the node, while in our approach an arc of $50^{\circ}$ is enough to achieve the same precision, therefore in a shorter flight time.

In contrast with the previous works, we propose a lightweight algorithm that can run even in very resourceconstrained embedded systems. Our algorithm is designed to maximize the accuracy with the minimal number of UWB range measurements, thereby reducing the UAV mission time. To the best of our knowledge, there is no previous work that combines UWB and WUR for enabling a drone to perform ground sensor node localization and optimizing for both accuracy and node energy consumption.

\section{Embedded Multi-lateration Algorithm}

Our algorithm focuses on a 2-dimensional (2D) use case, where all the sensor nodes are deployed on the ground at the same altitude. Even if less general than the 3-dimensional (3D) one, our use case is still representative for the majority of practical industrial application scenarios [16], [26]. Moreover, many key aspects of the 2D use case also apply to the $3 \mathrm{D}$ case.

\section{A. Background: UWB multi-lateration}

The purpose of a multi-lateration algorithm is to estimate the sensor node position with high accuracy, using way-points as input. A way-point is a structure of type $\left(x_{d}, y_{d}, r\right)$, where the pair $\left(x_{d}, y_{d}\right)$ represents the drone position (e.g., GPS position) [15], and $r$ is the range measurement from the drone to the node (i.e., ground projection in a 2-D plane). Apart from the ranging error, the measurements of GPS position and altitude are also affected by noise which consequently influences the accuracy of the overall multi-lateration estimation. More way-points lead to a more accurate estimation but also increases the number of transmitted packets and therefore the total energy consumption.

A common algorithm for fast multi-lateration is the RBMML [23], widely used in many localization systems [27] due to its closed-form solution. In a noise-free situation, the 


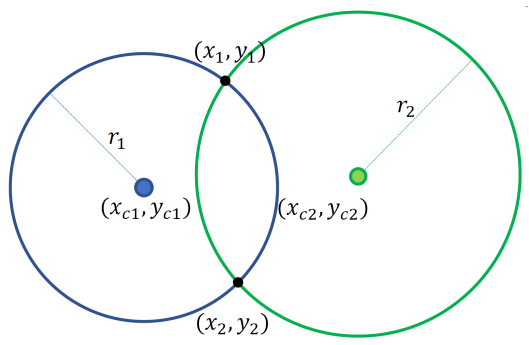

Fig. 2: Geometrical representation of two way-points.

coordinates of the unknown node and the $\mathrm{N}$ acquired waypoints $\left(x_{i}, y_{i}, r_{i}\right)$, with $i=1,2, \ldots N$, satisfy Equation 1 .

$$
\left(x_{i}-x\right)^{2}+\left(y_{i}-y\right)^{2}=r_{i}^{2}
$$

By subtracting the $\mathrm{N}$-th equation from the first $\mathrm{N}-1$ equations, it leads to a linear system of $\mathrm{N}-1$ equations, because the quadratic terms in $\mathrm{x}$ and $\mathrm{y}$ cancel out, and it can be solved in the least-squares sense. In the absence of any noise on the range distance measurements, RB-MML, as well as all other methods, returns the optimal node localization using only three way-points. Therefore, it requires a few simple matrix multiplications, but its accuracy drops quickly in real cases due to the inevitable noise on the measurements, as shown in Section V.

Assuming that the range measurements are affected by zero-mean Gaussian noise, the maximum likelihood estimation (MLE) is obtained by minimizing the cost function in Equation 2 [25]. In this way, the pair $(\tilde{x}, \tilde{y})$, which minimizes the cost function, is the solution that makes the range measurements "the most likely". Our optimization problem is represented by the non-convex and non-linear function in Equation 3. Choosing proper initialization values for $(x, y)$ is crucial to avoid local minima that might lead to high-cost values.

$$
\begin{gathered}
L(x, y)=\sum_{i=1}^{N}\left(\sqrt{\left(x_{i}-x\right)^{2}+\left(y_{i}-y\right)^{2}}-r_{i}\right)^{2} \\
(\tilde{x}, \tilde{y})=\arg \min L(x, y)
\end{gathered}
$$

\section{B. Embedded multi-lateration algorithm}

In our 2D case, the geometrical interpretation of a single way-point $\left(x_{1}, y_{1}, r_{1}\right)$, suggests that the sensor node is somewhere on the circumference of the circle with center in $\left(x_{1}, y_{1}\right)$ and radius $r_{1}$. With a second way-point, the number of possible locations for the node is reduced to two possibilities: the two points given by the intersection of the two circumferences, as shown in Figure 2. These two intersection points can be easily computed by solving the equation system described in [28]. In our approach, we first compute the cost function in Equation 2 for both the two candidate points, and we define as $\left(x_{0}, y_{0}\right)$ the one which leads to the smaller cost.

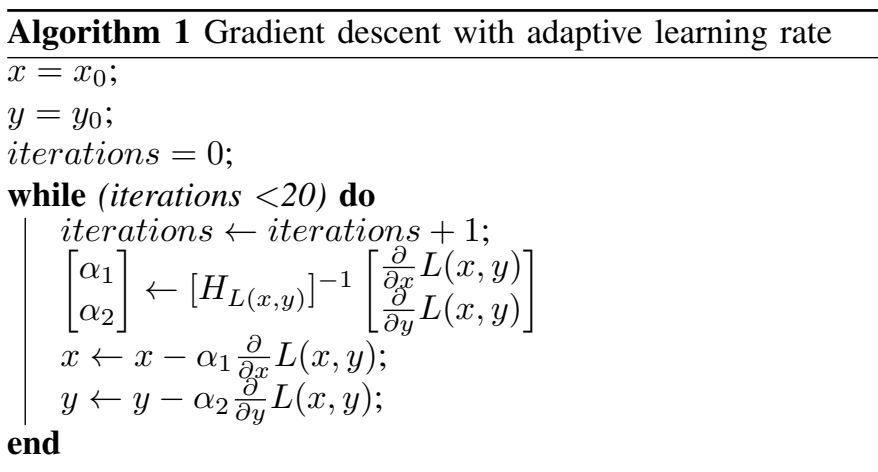

In an ideal situation, where the way-point elements have zero sensor noise, the solution $\left(x_{0}, y_{0}\right)$ matches the global minimum of the optimization problem from Equation 3 and leads to a zero cost. However, range measurements are affected by noise, whose standard deviation has an impact on how far $\left(x_{0}, y_{0}\right)$ is from the global minimum of the optimization problem. However, range measurements are performed using UWB, which has a precision of $10 \mathrm{~cm}^{1}$. Since the UWB distance measurements are in the range of meters, their sub$10 \mathrm{~cm}$ error practically results in having the $\left(x_{0}, y_{0}\right)$ solution located near to the global minimum ${ }^{2}$, and consequently, we use $\left(x_{0}, y_{0}\right)$ as the initialization value for our optimizer.

The optimizer we propose is a gradient descent algorithm meant to run in a low-resources embedded platform, and therefore we aim at minimizing the number of iterations until convergence. For this reason, we propose to use Newton's update rule to online adjust the learning rate of the algorithm with respect to the inverse of the Hessian matrix, as shown in Algorithm 1. With this choice, the optimizer converges faster in the first few iterations, and the learning rate decreases as they progress (on average from 0.5 to 0.001 ). Experimentally, we observed that Algorithm 1 achieves a difference of less than $1 \mathrm{~mm}$ between two consecutive iterations after less than 20 iterations. In this way, our algorithm is not data-dependent, and the iteration number - and also running time - is always constant and predictable (4 ms for an ARM Cortex M4 $168 \mathrm{MHz})$.

\section{System ARchitecture}

We designed the localization unit to be placed on the drone, and the sensor nodes deployed in the testing area (Figure 3). The localization unit manages the communication with the nodes, acquires the range measurements, performs the sensor node localization and sends trajectory commands to the drone. The overall system is closed-loop and all computation is carried by the MCU on the localization unit.

\footnotetext{
${ }^{1}$ https://www.decawave.com/sites/default/files/resources/ dw1000-datasheet-v2.09.pdf

${ }^{2}$ In our experiments, we observed that the optimization function, in the proximity of the global minimum, is always convex. Therefore, if the initialization value (i.e., $x_{0}, y_{0}$ ) lies in this convex region, the optimizer quickly converges to the global minimum. In our extensive evaluation (more than 1000 experiments), the optimizer always managed to find the global minimum, using the proposed initialization value.
} 

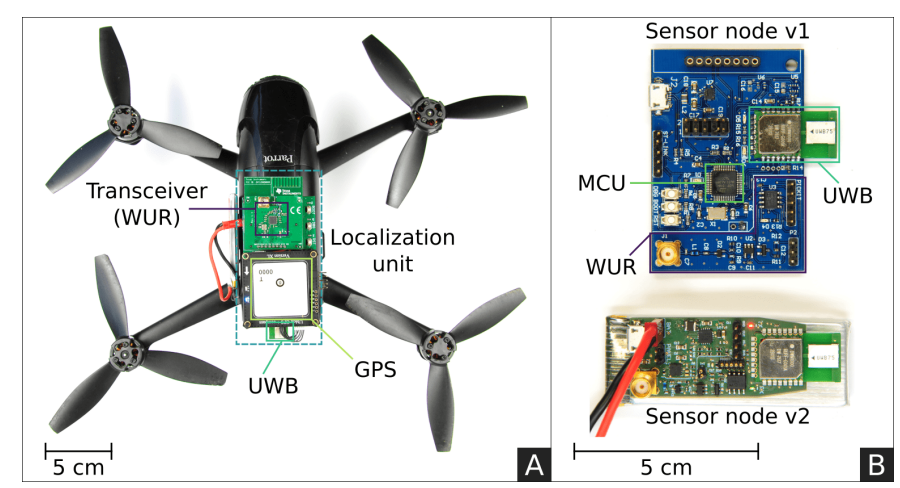

Fig. 3: A) Our prototype based on a COTS Parrot Bebop 2 extended with our localization unit. B) Sensor node, both v1 and v2 (optimized form factor).

The core of the sensor node is a STM32L433 MCU, which manages communications and sensors' acquisition; it has been chosen for its low sleep current consumption of just $50 \mathrm{nA}$. In particular, the MCU is in charge of controlling the UWB module in the ranging process as well as its power mode. The UWB module (Decawave DWM1000 ${ }^{3}$ ) is configured for the maximum transmit power and the smallest data-rate (i.e., $110 \mathrm{kbps}$ ) to maximize the operating range - over $150 \mathrm{~m}$ line of sight $(\mathrm{LoS})$. The sensor node is also capable of energy harvesting: a power management circuit, built around the BQ25570 from Texas Instruments ${ }^{4}$, together with a solar cell enables the node to charge the battery during the listening state and therefore, to achieve energy sustainability. The WUR unit is one of the key elements for energy-efficiency as it comes with nano-Watt power consumption [12]. The WUR used in this work has a sensitivity of down to $-55 \mathrm{~dB}$ while listening to wake-up messages. To increase energy-efficiency, we exploit the WUR to achieve asynchronous communication with the UWB module. The total power consumed by our sensor node during the listening state is $3.9 \mu \mathrm{W}$. In our application scenario, we assume that WUR is the only active element of the sensor node during listening. Once the data acquisition is completed, the node goes back in sleep mode. During the whole process of interaction with the drone, the sensor node consumes a total energy of $31 \mathrm{~mJ}$.

The localization unit operates on board the drone and it contains modules for position estimation, ranging and wakeup transmission. The GPS module ( $\mathrm{uBlox} M 8 \mathrm{~T}^{5}$ ) is used to determine the latitude and longitude of the UAV, while the high precision barometer (TE MS6511 ${ }^{6}$ ) provides altitude information. Even if the UAV has its own position estimator, the localization unit relies on its own sensors for more flexibility and control over the accuracy. The localization unit uses a

\footnotetext{
${ }^{3} \mathrm{https}: / /$ www.decawave.com/sites/default/files/resources/dwm1000datasheet-v1.3.pdf

${ }^{4}$ https://www.ti.com/document-viewer/BQ25570/datasheet/featuresslusbh25638\#SLUSBH25638

${ }^{5} \mathrm{https}: / /$ www.u-blox.com/sites/default/files/NEO-LEA-M8TFW3_DataSheet_\%28UBX-15025193\%29.pdf

${ }^{6}$ https://www.te.com/global-en/product-CAT-BLPS0036.html
}

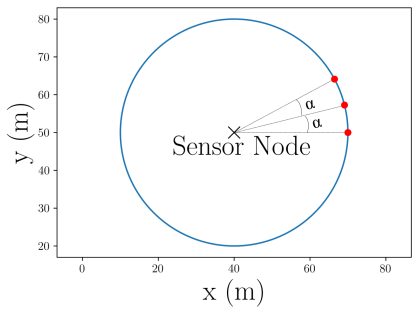

(a) Alpha angle is varied

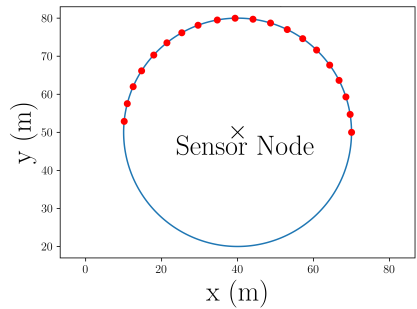

(b) Way-point density is varied
Fig. 4: The red spots indicate the location where the drone acquires a new way-point.

radio transceiver (TI CC1200 ${ }^{7}$ ) to emit wake-up beacons for the WUR, while the UAV is flying to wake up the sensors which are located in its range. The WUR receives the wakeup in a range of $35 \mathrm{~m}$.

\section{EXPERIMENTAL RESUlts}

This section presents the experimental results and analysis of the embedded multi-lateration algorithm presented in Section III. The evaluation is performed with both synthetic data and experimental UWB measurements collected flying with our prototype drone. In the ideal case of UWB measurements affected by Gaussian noise with zero-mean, the proposed algorithm would lead to the maximum likelihood estimate, due to the minimization of our cost function (see Section III). Our synthetic data are generated by altering the range measurements by zero-mean Gaussian noise $\left(\sigma_{r}=10 \mathrm{~cm}\right.$, according to the UWB module datasheet), leaving the waypoints location unaltered. However, real-world measurements are characterized by non-Gaussian noise, due to factors like non-line-of-sight conditions or antenna delays, which can have a significant impact on the overall localization accuracy. In this case, the drone's position, acquired every time a new waypoint is visited, is obtained from the onboard localization unit.

In the rest of this section, we refer to the way-point structure $\left(x_{d}, y_{d}, r\right)$, where $x_{d}$ and $y_{d}$ are calculated by converting GPS latitude and longitude, into Cartesian coordinates (east-northup coordinate system). The ground distance - i.e., the 3D projection on the ground of the UWB range measurement is calculated as $r=\sqrt{z^{2}-a^{2}}$, where $z$ is the UWB range measurement, and $a$ is the altitude provided by the onboard barometer.

\section{A. Algorithm evaluation on synthetic data}

In the first experiment, Figure $4 \mathrm{a}$, the node position is estimated via multi-lateration performing an acquisition procedure where the drone visits three different way-points, on a circular trajectory, and it acquires a new measurement for each of them. The angle between two consecutive way-points and the node position is defined as the alpha angle $(\alpha)$, which can always be represented as a central angle due to the selected trajectory. In our experiment configuration, we always enforce two equal alpha angles by selecting the visited way-points.

\footnotetext{
${ }^{7}$ https://www.ti.com/document-viewer/CC1200/datasheet
} 


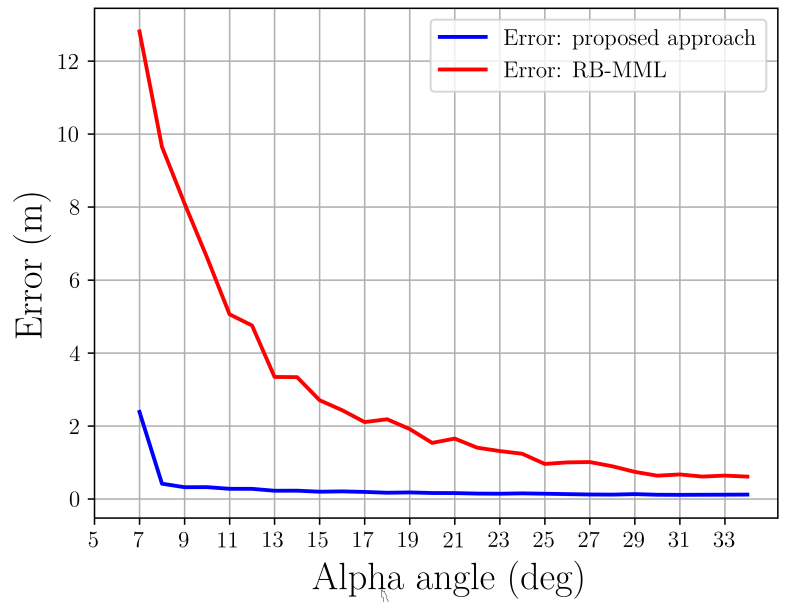

Fig. 5: Localization error as function of alpha angle $\left(\alpha_{1}=\alpha_{2}\right)$, for the configuration shown in Figure 4a.

In Figure 5, we show the mean localization error - as a function of the alpha angles - over 300 acquisition procedures, for every angle in the range $7^{\circ}-34^{\circ}$ with an incremental step of $2^{\circ}$. This localization error is evaluated for both the proposed approach and the RB-MML algorithm. Results highlight how our approach outperforms the RB-MML baseline, with a reduction of $\sim 6 \times$ of the peak error $\left(\alpha=7^{\circ}\right)$ and minimum error for the proposed algorithm as low as $12 \mathrm{~cm}$ vs. $58 \mathrm{~cm}$ for RB-MML. Considering a threshold error of $2 \mathrm{~m}$, our approach and RB-MML can met this requirement with an alpha angle of $7^{\circ}$ and $19^{\circ}$, respectively, meaning RB-MML would require $\sim 3 \times$ longer flight distance ${ }^{8}$.

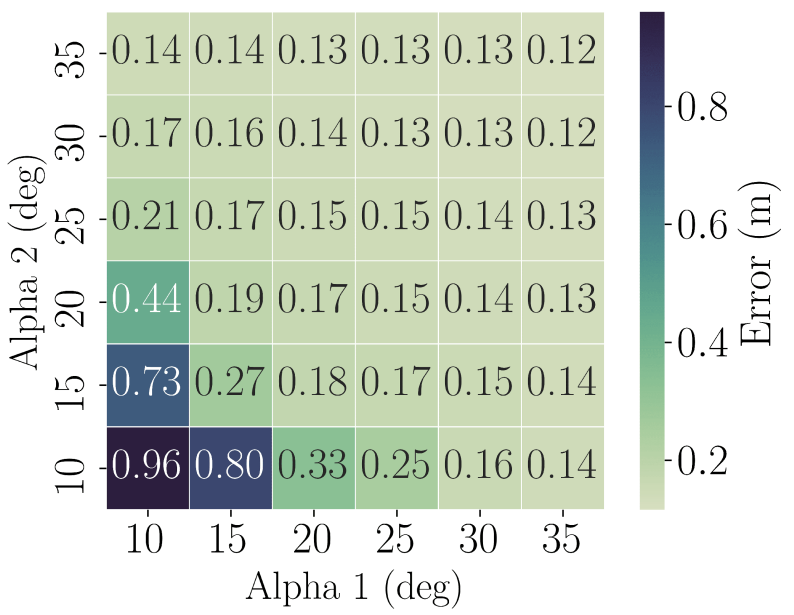

Fig. 6: Localization error as a function of the two alpha angles.

In Figure 6, we present a second experiment based on the configuration shown in Figure 4a, but this time we explore the localization trade-off of different alpha angles. Results demonstrate how the localization error depends on both angles, and how it can be significantly reduced, increasing them. We

\footnotetext{
${ }^{8} \mathrm{We}$ assume a ranging radius of $10 \mathrm{~m}$.
}

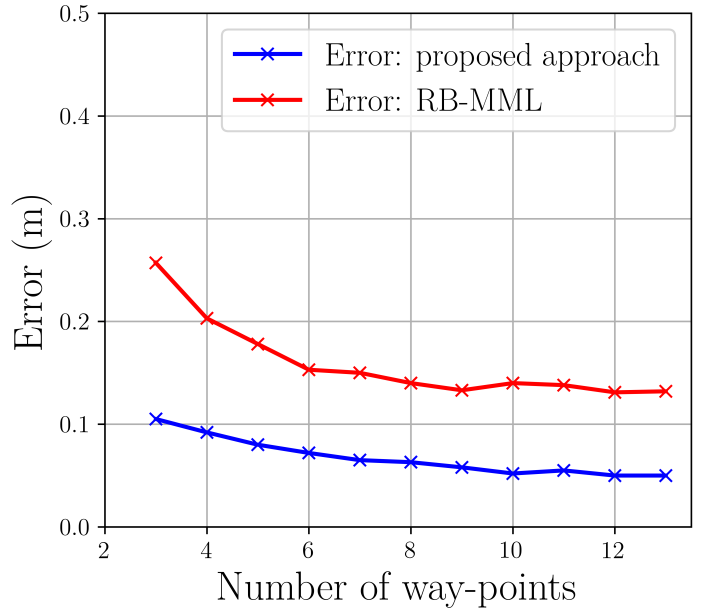

Fig. 7: Localization error as function of the way-point number, for the configuration shown in Figure $4 \mathrm{~b}$.

can also observe that the error is equally dependent on both alpha angles, being almost constant along the main diagonal and its parallels (i.e., from top-left to bottom-right corner).

In Figure 7, we report the mean localization error as a function of the number of way-points, considering them uniformly distributed on the semi-circumference of a circular trajectory, as shown in Figure 4b. Also in this case, our proposed multilateration approach performs on average $2 \times$ better than RBMML. Considering the highest number of way-points analyzed in this evaluation - i.e., 13 way-points -, Figure 7 shows a reduction of $40 \%$ on the mean error compared to the minimum of 3 way-points. Such an improvement comes at the price of increased UWB activity and flight time due to the additional way-points. For this reason, we choose to use three way-points as a good trade-off between accuracy and consumed energy.

\section{B. Algorithm evaluation with a flying drone}

In this section, we aim to confirm, with in-field experiments, the insights and system's properties highlighted with the synthetic data analysis (Section V-A). For this aim, we use our drone prototype - i.e., a Parrot Bebop 2 quadrotor extended with our onboard custom localization unit - on a grass-field mission area of $40 \times 40 \mathrm{~m}$. The origin of the Cartesian system of the multi-lateration algorithm corresponds to the take-off point, from which the drone flies at a constant $15 \mathrm{~m}$ altitude. The sensor nodes are randomly distributed in the mission area at unknown locations - by the drone. Their Cartesian coordinates are recorded to be used as ground-truth for the localization error calculation. The drone position is provided by the aboard GPS module, representing an additional source of error - root-mean-squared-error of $0.6 \mathrm{~m}$ - compared to the ideal synthetic data scenario.

The drone flight policy was set to use three way-points and enforcing two equal alpha angles $\left(\alpha_{1} \approx \alpha_{2}\right)$. As expected, the localization error is higher for small alpha angles, up to $3.7 \mathrm{~m}$ for angles below $15^{\circ}$, but decreases to a mean error lower than $1 \mathrm{~m}$ for angles higher than $20^{\circ}$. Pushing our system to its 
limit, we achieved an in-field mean localization error of only $0.6 \mathrm{~m}$ (peaking at $1.1 \mathrm{~m}$ ) with $40^{\circ}$ alpha angles. However, the localization error does not further improve for higher angles because the GPS uncertainty lower bounds it.

\section{Conclusions}

This paper proposes the combination of emerging communication technologies, such as UWB and WUR, to develop a novel localization system that enables mobile robots, such as drones, to precisely localize wireless sensor nodes whose positions are not known a priori. In our scenario, a drone acts as a mobile gateway of the WSN, and it uses a multilateration algorithm to localize the sensor nodes. We demonstrate our system's capability with a closed-loop, fully-working prototype, where our custom electronics - both onboard a COTS drone and deployed on the mission area as sensor nodes - works in combination with the proposed localization algorithm. The approach we propose is suitable for many industrial application scenarios where a mobile robot has to localize in its surrounding imprecisely positioned objects, followed by an energy-efficient data acquisition phase.

Our in-field evaluation shows how the proposed multilateration algorithm can overtake SoA accuracy with a submeter localization error, being computationally inexpensive - i.e., the entire localization procedure runs in $4 \mathrm{~ms}$ on the Cortex-M4 MCU aboard the drone. At the same time, by using the minimum number of three range measurements, we keep the UWB energy consumption in the sensor node as small as only $31 \mathrm{~mJ}$ - during the whole localization-acquisition process.

\section{REFERENCES}

[1] L. Yang, Q. Sun, and Z.-S. Ye, "Designing mission abort strategies based on early-warning information: Application to uav," IEEE Transactions on Industrial Informatics, 2019.

[2] D. Palossi, A. Gomez, S. Draskovic, A. Marongiu, L. Thiele, and L. Benini, "Extending the lifetime of nano-blimps via dynamic motor control," Journal of Signal Processing Systems, vol. 91, no. 3-4, pp. 339-361, 2019.

[3] D. Palossi, F. Conti, and L. Benini, "An open source and open hardware deep learning-powered visual navigation engine for autonomous nanouavs," in 2019 15th International Conference on Distributed Computing in Sensor Systems (DCOSS). IEEE, 2019, pp. 604-611.

[4] I. Jawhar, N. Mohamed, J. Al-Jaroodi, and S. Zhang, "An architecture for using autonomous underwater vehicles in wireless sensor networks for underwater pipeline monitoring," IEEE Transactions on Industrial Informatics, vol. 15, no. 3, pp. 1329-1340, 2018.

[5] D. Wu, D. Chatzigeorgiou, K. Youcef-Toumi, and R. Ben-Mansour, "Node localization in robotic sensor networks for pipeline inspection," IEEE Transactions on Industrial Informatics, vol. 12, no. 2, pp. 809819,2015

[6] M. Magno, D. Boyle, D. Brunelli, E. Popovici, and L. Benini, "Ensuring survivability of resource-intensive sensor networks through ultra-low power overlays," IEEE Transactions on Industrial Informatics, vol. 10, no. 2, pp. 946-956, 2013.

[7] S. Saraswat, A. Tripathi, H. P. Gupta, and T. Dutta, "An energy efficient monitoring system for precision agriculture in wireless sensor networks," in 10th International Conference on Advances in Science, Engineering and Technology (ICASET-18), 2018.

[8] M. Farooq-I-Azam, Q. Ni, and E. A. Ansari, "Intelligent energy efficient localization using variable range beacons in industrial wireless sensor networks," IEEE Transactions on Industrial Informatics, vol. 12, no. 6, pp. 2206-2216, 2016
[9] O. Saukh, D. Hasenfratz, and L. Thiele, "Reducing multi-hop calibration errors in large-scale mobile sensor networks," in Proceedings of the 14th International Conference on Information Processing in Sensor Networks. ACM, 2015, pp. 274-285.

[10] H. Huang and A. V. Savkin, "An algorithm of reactive collision free 3d deployment of networked unmanned aerial vehicles for surveillance and monitoring," IEEE Transactions on Industrial Informatics, 2019.

[11] K. Minne, N. Macoir, J. Rossey, Q. Van den Brande, S. Lemey, J. Hoebeke, and E. De Poorter, "Experimental evaluation of uwb indoor positioning for indoor track cycling," Sensors, vol. 19, no. 9, p. 2041, 2019.

[12] M. Magno, V. Jelicic, B. Srbinovski, V. Bilas, E. Popovici, and L. Benini, "Design, implementation, and performance evaluation of a flexible low-latency nanowatt wake-up radio receiver," IEEE Transactions on Industrial Informatics, vol. 12, no. 2, pp. 633-644, 2016.

[13] J. Tiemann and C. Wietfeld, "Scalability, real-time capabilities, and energy efficiency in ultra-wideband localization," IEEE Transactions on Industrial Informatics, vol. 15, no. 12, pp. 6313-6321, 2019.

[14] B. J. Silva and G. P. Hancke, "Ranging error mitigation for throughthe-wall non-line-of-sight conditions," IEEE Transactions on Industrial Informatics, 2020.

[15] F. B. Sorbelli, S. K. Das, C. M. Pinotti, and S. Silvestri, "Range based algorithms for precise localization of terrestrial objects using a drone," Pervasive and Mobile Computing, vol. 48, pp. 20-42, 2018.

[16] _ " "On the accuracy of localizing terrestrial objects using drones," in 2018 IEEE International Conference on Communications (ICC). IEEE, 2018, pp. $1-7$.

[17] H. Li and A. V. Savkin, "Wireless sensor network based navigation of micro flying robots in the industrial internet of things," IEEE Transactions on industrial informatics, vol. 14, no. 8, pp. 3524-3533, 2018.

[18] M. Golestanian, H. Lu, C. Poellabauer, and J. Kenney, "Rssi-based ranging for pedestrian localization," in 2018 IEEE 88th Vehicular Technology Conference (VTC-Fall). IEEE, 2018, pp. 1-5.

[19] S. Kumar, S. Gil, D. Katabi, and D. Rus, "Accurate indoor localization with zero start-up cost," in Proceedings of the 20th annual international conference on Mobile computing and networking, 2014, pp. 483-494.

[20] E. Dahlgren and H. Mahmood, "Evaluation of indoor positioning based on bluetooth smart technology," Master's thesis, 2014.

[21] M. Siekkinen, M. Hiienkari, J. K. Nurminen, and J. Nieminen, "How low energy is bluetooth low energy? comparative measurements with zigbee/802.15. 4," in 2012 IEEE wireless communications and networking conference workshops (WCNCW). IEEE, 2012, pp. 232-237.

[22] J. H. An and L. Choi, "Inverse fingerprinting: server side indoor localization with bluetooth low energy," in 2016 IEEE 27th Annual International Symposium on Personal, Indoor, and Mobile Radio Communications (PIMRC). IEEE, 2016, pp. 1-6.

[23] J. Xu, J. He, Y. Zhang, F. Xu, and F. Cai, "A distance-based maximum likelihood estimation method for sensor localization in wireless sensor networks," International Journal of Distributed Sensor Networks, vol. 12, no. 4, p. 2080536, 2016.

[24] Y. Zhou, "A closed-form algorithm for the least-squares trilateration problem," Robotica, vol. 29, no. 3, pp. 375-389, 2011.

[25] M. Larsson, V. Larsson, K. Astrom, and M. Oskarsson, "Optimal trilateration is an eigenvalue problem," in ICASSP 2019-2019 IEEE International Conference on Acoustics, Speech and Signal Processing (ICASSP). IEEE, 2019, pp. 5586-5590.

[26] A. Ledergerber, M. Hamer, and R. D'Andrea, "Angle of arrival estimation based on channel impulse response measurements," in 2019 IEEE/RSJ International Conference on Intelligent Robots and Systems (IROS). IEEE, 2019, pp. 6686-6692.

[27] J. Du, J.-F. Diouris, and Y. Wang, "A rssi-based parameter tracking strategy for constrained position localization," EURASIP Journal on Advances in Signal Processing, vol. 2017, no. 1, pp. 1-10, 2017.

[28] J. Li, X. Yue, J. Chen, and F. Deng, "A novel robust trilateration method applied to ultra-wide bandwidth location systems," Sensors, vol. 17, no. 4, p. 795, 2017. 